\title{
Risk aversion and effort under an incentive pay scheme with multiplicative noise Theory and experimental evidence
}

\author{
Nikolay Zubanov \\ Faculty of Economics and Business Administration, Goethe University \\ Frankfurt, Frankfurt am Main, Germany
}

\begin{abstract}
Purpose - The purpose of this paper is to consider the influence of individual risk preferences on the effectiveness of incentive pay schemes, by examining the link between individual effort and risk aversion in situations where outcome uncertainty multiplies with effort. Such "multiplicative noise" situations are common, occurring whenever payment is awarded per success rather than per attempt. Design/methodology/approach - The paper develops a theoretical model which predicts a negative risk aversion-effort link under multiplicative noise without a performance target (PT), and a weaker negative link once the target is introduced. This model is then taken to the data from a lab experiment where participants were randomly assigned to a control group, which received fixed pay, and a treatment group, which received a piece rate awarded with a certain probability, with and without a PT. Risk aversion is measured with a menu of lottery choices offered at the end of the experiment.

Findings - Compared to their peers in the control group, the more risk-averse participants in the treatment group put in progressively less effort in the absence of a PT. The introduction of a PT substantially weakens this negative risk aversion-effort link, so that there are no more significant differences in performance between the more and the less risk averse.

Research limitations/implications - The paper's findings speak to the empirical puzzles of incentive pay schemes backfiring and of the proliferation of PTs. The negative risk aversion-effort link may be one reason behind the failure of incentive schemes to deliver improved performance, whereas the weakening of this link may be one justification for the existence of PTs.

Practical implications - In the multiplicative noise environments, managers should take their workers' risk preferences into account when designing incentive pay schemes. A PT may be a useful motivational tool for the risk-averse workers who are more likely to under-perform.

Originality/value - The multiplicative noise environment has been largely overlooked by the existing literature, yet it is common in practice. An example is the work of a sales agent who receives a bonus per sales which succeeds with a certain probability after each customer contact. This paper is one of the first to model, and test experimentally, worker performance in this environment.

Keywords Employee motivation, Work performance and productivity,

Promotion and compensation, Labour economics

Paper type Research paper

\section{Introduction}

Consider a sales agent who earns a commission per deal which succeeds with a certain probability after each customer contact. The more contacts he or she makes, the higher are the expected earnings, but so too is the earnings risk. What is the relationship

The author thanks seminar participants at Goethe University Frankfurt, Erasmus University Rotterdam, Tilburg University and St. Petersburg University, and in particular Vladimir Karamychev, Floris Zoutman, Casper de Vries, Canice Prendergast and Bram Cadsby, for their helpful comments. Excellent assistance of Sabine van Boxtel, Marijn Matthijsse and Alvaro Serrano Macon in preparing the experiment featured in this study is gratefully acknowledged.
\end{abstract}


between the agent's risk aversion and the number of customer contacts he or she will choose to make under a given commission schedule? Suppose now that there is an individual sales target, falling below which the agent in question will not receive commissions. Will such a target affect the agent's effort choice?

The questions raised above go beyond the sales agent example. Risk preferences have been known to affect workers' responses to incentives and, by implication, the effectiveness of incentive pay schemes - a staple HR practice. Because more risk-averse workers require higher fixed compensation for a given exposure to risk (Prendergast, 2000), firms tend to offer lower piece rates to such workers (Lazear and Gibbs, 2009, p. 289). As a result, their response to incentives will be weaker. Although less risk-averse workers tend to dominate in incentive pay jobs (Bellemare and Shearer, 2010; Grund and Sliwka, 2010), since the sorting of people into jobs is never perfect, workers with varying risk preferences will still be observed in jobs giving the same exposure to financial risk, and may differ in the chosen effort level. This difference is a potential source of inefficiency: because a firm will have to design a uniform incentive contract to satisfy every worker's participation constraint, some workers will be enjoying a rent at the firm's expense.

The research on the risk aversion-effort link under a given incentive pay contract has been informed by two theoretical perspectives. One, based on expectancy theory (Vroom, 1964), predicts a negative relationship, as greater aversion to financial risk weakens "expectancy" - the perceived link between effort and performance outcome, thus reducing the motivation to exert effort. The other perspective, which stems from agency theory, distinguishes in its predictions between the following two cases. The first, which may be labelled "additive noise," is the classical case (Holmstrom and Milgrom, 1987; Baker, 1992) of the noise to output being additive to, and independent of, the product of a worker's effort. In this case, the risk aversion- effort link is zero or positive, depending on the configuration of the utility and costs of effort functions (Sloof and van Praag, 2010). The second is the less frequently studied "multiplicative noise" case, a label describing situations when the noise to output changes regularly with effort. I will proceed with this case in the rest of the paper.

The sales agent story in the beginning of the paper is a good example of multiplicative noise. Indeed, given the probability of success per contact $p$, the noise in the agent's earnings increases with the effort measured as the number of customer contacts $n$, with its variance becoming $n p(1-p)$. Intuitively, all else equal, more riskaverse agents, and in particular those with a greater dislike for earnings uncertainty, will face higher marginal costs of effort and will therefore put in less of it. This theoretical prediction was anticipated in Baker and Jorgensen (2003) and Sloof and van Praag (2008), and will be further developed here.

Consistent with the theory, the existing empirical research tends to find a negative correlation between individual risk aversion and expectancy or actual performance in situations resembling multiplicative noise. Examples include field studies of sales force compensation - the line of work where the multiplicative noise case is likely to apply (Pappas and Flaherty, 2006; Lo et al., 2011; Coughlan and Joseph, 2012), as well as laboratory experiments on differential responses to incentives by Cadsby et al. $(2007,2009)$ [1]. However, because these experimental studies incentivized performance through simple piece rate, the element of multiplicative noise was introduced through the uncertainty in the time it takes to do one task (an anagram), which is rather indirect. In my experiment, I introduce multiplicative noise directly, through awarding a piece rate per task with a certain probability, which is arguably a "cleaner" design. 
Turning to performance targets (PTs), the literature has viewed them mainly as "pacers to keep the sales force on track to achieve annual sales quotas" (Chung et al., 2014) or as an approximation of the nonlinear optimal incentive pay contract (Basu et al., 1985; Raju and Srinivasan, 1996). However, it may be argued within the agency theory that, by introducing the possibility of losing part of expected earnings, the targets increase the marginal product of effort to try to avoid this possibility. Because this increase is higher for underperforming, risk-averse workers, since they run a higher risk of losing their commission, the extra effort they will put in will be higher. As a result, the negative risk aversion-effort link will flatten out. The experimental finding in Gaba and Karla (1999), that workers were more likely to engage in high-risk, high stakes activities when PTs were set at a higher level, may be interpreted as supporting the above argument, albeit indirectly.

My study contributes to the relevant literatures by performing a direct experimental test of the risk aversion-effort link under multiplicative noise with and without PTs. My experiment involved 85 student participants performing a search task in two consecutive rounds. In the control group, a fixed pay was given in each round, while the treatment group received a piece rate with a given probability, without and with a PT in the first and second rounds, respectively. At the end of the experiment, each participant's risk aversion was measured using the Holt and Laury's (2002) metric. I find a negative relationship between risk aversion and effort in the treatment group in round 1 (no PT) and no relationship in round 2 (with PT). The control group shows no link in either round. Taken together, my results imply that piece rate incentives with multiplicative noise induce risk-averse workers to economize on effort to try to reduce their exposure to financial risk, and that introducing a PT weakens this tendency.

The rest of the paper is organized as follows. The next section presents a simple model of incentive pay with multiplicative noise to effort, deriving the relationships between risk aversion and optimal effort without and with a PT, and illustrating them numerically. Section 3 describes the experiment to test this model, and Section 4 reports some preliminary findings. Section 5 discusses the relevant empirical estimation issues. Section 6 presents the regression results. Section 7 discusses the results, relating them to the existing literature and practice of personnel management, and concludes.

\section{Theory and hypotheses}

Consider a worker doing a standard repetitive task, such as the work of a sales agent given as an example in the introduction, with each attempt having a certain probability of success $\bar{\gamma}$. With a fixed wage $B$ and a commission per each successfully completed task normalized to 1 , the worker's total earnings are $B+\gamma x$, where $x$ is the number of attempts and $\gamma \in[0,1]$ is the empirical success frequency. The fluctuations of $\gamma$ around its expected value $\bar{\gamma}$ introduce noise to output which is multiplicative to worker's effort, since the variance of earnings is $\bar{\gamma}(1-\bar{\gamma}) x$. The worker can choose any $x$ starting from a certain minimum $x_{0}$. The firm can only observe and enforce the minimum effort level $x_{0}$, but not any effort above it; therefore, the firm contracts on output rather than effort.

As in Holt and Laury (2002), Cadsby et al. (2007, 2009), Sloof and van Praag (2008) and many other studies, we assume the worker to have an exponential utility function [2] which is increasing and concave in total earnings. We further assume, for analytical convenience, that the worker's utility is separable in the total earnings and effort costs. The costs of effort, $c(x)$, is a monotonically increasing and convex function of effort. 
Approximating the discrete distribution of the success frequency $\gamma$ with a continuous one for large $x$, the worker's expected utility net of effort costs is:

$$
U(x)=\int_{0}^{1} \frac{1-e^{-\delta(B+\gamma x)}}{\delta} d F(\gamma)-c(x), \quad c^{\prime}(x)>0, \quad c^{\prime \prime}(x)>0
$$

Here $\delta$ is the Arrow-Pratt measure of risk aversion (higher $\delta$ means greater risk aversion, negative $\delta$ means risk loving) and $F(\gamma)$ is the cumulative distribution function of $\gamma$, which converges to normal with mean $\bar{\gamma}$ and standard deviation $\sqrt{\bar{\gamma}(1-\bar{\gamma}) / x}$ as $x$ becomes large. I further assume that the costs of effort function $c(x)$ is steep enough for the optimal level of effort $x^{*}<\infty$, maximizing (1), to exist.

It is convenient to "standardize" success frequency $\gamma$, using:

$$
z=\sqrt{x} \frac{\gamma-\bar{\gamma}}{\sqrt{\bar{\gamma}(1-\bar{\gamma})}}
$$

instead of $\gamma$ in the utility function (1). Then $F(\gamma)=\Phi(z)$, where $\Phi(\cdot)$ is the cumulative standard normal distribution function. The utility function (1) redefined in terms of $z$ becomes:

$$
U(x)=\int_{-\infty}^{\infty} \frac{1-e^{-\delta(B+z \sqrt{x \bar{\gamma}(1-\bar{\gamma})}+\bar{\gamma} x)}}{\delta} d \Phi(z)-c(x)
$$

The optimal effort level $x^{*}$ maximizing (2) is determined from the following first-order condition:

$$
U^{\prime}(x)=\int_{-\infty}^{\infty} e^{-\delta\left(B+z \sqrt{x^{*} \bar{\gamma}(1-\bar{\gamma})}+\bar{\gamma} x^{*}\right)}\left(\frac{z \sqrt{\bar{\gamma}(1-\bar{\gamma})}}{2 \sqrt{x^{*}}}+\bar{\gamma}\right) d \Phi(z)-c^{\prime}\left(x^{*}\right)=0
$$

Differentiating $x^{*}$ in (3) with respect to the risk aversion parameter $\delta$ gives:

$$
\frac{d x^{*}}{d \delta}=-\frac{\int_{-\infty}^{\infty} e^{-\delta\left(B+z \sqrt{x^{*} \bar{\gamma}(1-\bar{\gamma})}+\bar{\gamma} x^{*}\right)}\left(\frac{z \sqrt{\bar{\gamma}(1-\bar{\gamma})}}{2 \sqrt{x^{*}}}+\bar{\gamma}\right)\left(B+z \sqrt{x^{*} \bar{\gamma}(1-\bar{\gamma})}+\bar{\gamma} x^{*}\right) d \Phi(z)}{\int_{-\infty}^{\infty} e^{-\delta\left(B+z \sqrt{x^{*} \bar{\gamma}(1-\bar{\gamma})}+\bar{\gamma} x^{*}\right)}\left[\delta\left(\frac{z \sqrt{\bar{\gamma}(1-\bar{\gamma})}}{2 \sqrt{x^{*}}}+\bar{\gamma}\right)^{2}+\frac{z \sqrt{\bar{\gamma}(1-\bar{\gamma})}}{4 x^{*} \sqrt{x^{*}}}\right] d \Phi(z)+c^{\prime \prime}(x)}
$$

The sign of $d x^{*} / d \delta$ in (4) is always negative. Hence, my first hypothesis:

H1. For a given incentive pay contract with multiplicative noise, optimal effort $x^{*}$ decreases with risk aversion.

Intuitively, in an incentive pay model where the noise to output is multiplicative in effort, the optimal effort level should decrease with risk aversion because higher effort creates extra uncertainty, which is the more costly to bear the more risk-averse a worker is. In contrast, when the noise is additive to, and independent of, effort, the uncertainty it creates is independent of the worker's actions. In fact, any additive and independent noise component to the utility function (2) can be separated from effort by factoring it out of the integral, and thus will not affect the first-order conditions determining $x^{*}$. Sloof and van Praag (2008, pp. 796-798) provide analytical results supporting this statement. 
$2.1 P T$

With the PT, the worker receives fixed wage plus commissions, $B+\gamma x$, if the number of successes is at or above a certain minimum output level $y_{0}>0$, that is, when:

$$
\gamma x=z \sqrt{x \bar{\gamma}(1-\bar{\gamma})}+\bar{\gamma} x \geqslant y_{0}
$$

Failing this, he or she receives only fixed wage $B$. The worker's expected utility function then becomes:

$$
\begin{aligned}
U^{P T}(x) & =\int_{-\infty}^{z_{0}(x)} \frac{1-e^{-\delta B}}{\delta} d \Phi(z)+\int_{z_{0}(x)}^{\infty} \frac{1-e^{-\delta(B+z \sqrt{x \bar{\gamma}(1-\bar{\gamma})}+\bar{\gamma} x)}}{\delta} d \Phi(z)-c(x) \\
& =U(x)-A(x)
\end{aligned}
$$

where $z_{0}(x)=\left(y_{0}-\bar{\gamma} x\right) / \sqrt{x \bar{\gamma}(1-\bar{\gamma})}$ is the minimum of the standardized success frequency at which PT is met given the level of effort $x$, and the term:

$$
A(x)=\int_{-\infty}^{z_{0}(x)} \frac{e^{-\delta B}-e^{-\delta(B+z \sqrt{x \bar{\gamma}(1-\bar{\gamma})}+\bar{\gamma} x)}}{\delta} d \Phi(z)
$$

is the expected utility loss from failing to meet PT despite trying.

Three facts are true about $A(x)$ :

(1) $A(x)>0$ for all $x_{0} \leqslant x<x<\infty$, since for these values of $x$ the expression under the integral sign in (6) is positive.

(2) $\lim _{x \rightarrow \infty} \mathrm{A}(x)=0$, since $\lim _{x \rightarrow \infty} z_{0}(x)=-\infty$.

(3) $A(x)$ is monotonic in $x$ as long as the probability of meeting PT increases with $x$ at a faster rate than does the disutility of effort. This happens whenever the derivative of the cumulative distribution function $\Phi(z)$ at $z=z_{0}(x)$ is greater than $\left(e^{-\delta B}-e^{-\delta\left(B+y_{0}\right)}\right) / \delta$ (the value of the expression under integral in (6) at $z=z_{0}(x)$ ), which happens in particular when $y_{0}$ is close to the expected number of successes given the effort without PT, $x^{*} \bar{\gamma}$.

These three facts taken together imply that $A^{\prime}(x)<0$ and $A^{\prime \prime}(x)>0$. Given (5), $A^{\prime}(x)<0$ further implies that for the optimal effort level without PT, $x^{*}$, derived from (3), $U^{\prime}(x)^{*}$ $-A^{\prime}(x)^{*}=-A^{\prime}(x)^{*}>0$. Therefore, the optimal level of effort with PT, $x_{m}^{*}$, will exceed $x^{*}$. Finally, given that $A^{\prime}\left(x_{m}^{*}\right)=-\left(U^{\prime}\left(x_{m}^{*}\right)-U^{\prime}\left(x^{*}\right)\right)$, the convexity and monotonicity of $A(x)$ implies that the difference between $x_{m}^{*}$ and $x^{*}$ decreases with $x^{*}$, that is:

$$
\frac{d\left(x_{m}^{*}-x^{*}\right)}{d x^{*}}=\frac{\frac{d x_{m}^{*}}{d \delta}-\frac{d x^{*}}{d \delta}}{\frac{d x^{*}}{d \delta}}<0
$$

Recalling from (4) that $d x^{*} / d \delta<0$, inequality (7) implies:

$$
\frac{d x_{m}^{*}}{d \delta}-\frac{d x^{*}}{d \delta}>0
$$


Hence the second hypothesis:

H2. For a given incentive pay contract without PT, the introduction of PT will weaken the negative relationship between effort and risk aversion.

For the intuition behind $H 2$, consider how the introduction of PT changes the levels of effort previously chosen by the worker HL with low risk aversion and high effort, and the worker LH with high risk aversion and low effort. For the hard-working HL, there is almost no change, since his effort practically guaranties meeting PT. On the other hand, the relatively low effort that LH used to put in exposes him to a higher expected utility loss $A(x)$ from missing the target, compelling him to increase effort to try to avoid this loss.

It must be stressed that $H 2$ will apply only to the workers who still choose to participate in the contract when PT is introduced. In the case of the optimal, profitmaximizing incentive pay contract without PT, the participation constraint will be binding for the least productive worker who will make the minimum acceptable effort $x_{0}$. As PT is introduced, this worker and those near him in effort (the cut-off effort level depends on $y_{0}$ ) will pull out of the contract, since for these workers the utility from participation will fall below their reservation utility by the amount $A(x)>0$. Thus, similar to the incentive and sorting effects established by Lazear (2000) for the additive noise case, the introduction of PT is expected to result in: the departure of the least productive workers; and an increase in the effort by the workers for whom it is still profitable to remain in the firm. However, since the participants were required, and agreed, to stay for the entire duration of my experiment, I will abstract from PT's effect on participation in what follows.

\subsection{Numerical solutions for optimal effort}

This section illustrates my hypotheses with numerical solutions for the worker's optimal effort levels $x^{*}$ (without PT) and $x_{m}^{*}$ (with PT) as functions of risk aversion[3]. The parameters of the utility functions (2) and (5), underlying the solutions, are listed in Table I. The values of the probability of success $\bar{\gamma}$, fixed wage $B$ and the minimum acceptable effort and output requirement, $x_{0}$ and $y_{0}$, respectively, are the same as in the experiment (next Section 3). The range of the risk aversion parameter $\delta$ is restricted to be above 0 , to guarantee the existence of an optimal solution given the parameters of the cost function. These parameters have been chosen so that the optimal solution without PT, $x^{*}$, corresponding to the average risk aversion level in the experiment's treatment group is close to its empirical counterpart.

Figure 1 plots logarithms of optimal effort levels without and with PT as functions of risk aversion calculated from their respective first-order conditions given the

\begin{tabular}{|c|c|c|}
\hline Parameter & Value & \\
\hline The probability of success, $\bar{\gamma}$ & 0.25 & \\
\hline Cost function: $c(x)=a e^{b x}$ & & Table I. \\
\hline$a$ & $10^{-6}$ & Parameters \\
\hline$b$ & 0.35 & underlying numerical \\
\hline Fixed wage, $B$ & 0 & solutions for optimal \\
\hline Performance target, $y_{0}$ & 8 & effort levels without \\
\hline Minimum acceptable effort, $x_{0}$ & 0 & and with \\
\hline Risk aversion parameter, $\delta$ & {$[0,1]$} & performance target \\
\hline
\end{tabular}




\section{6}

Figure 1.

The theoretical link between log optimal effort and risk aversion with and without performance target $(\mathrm{PT})$

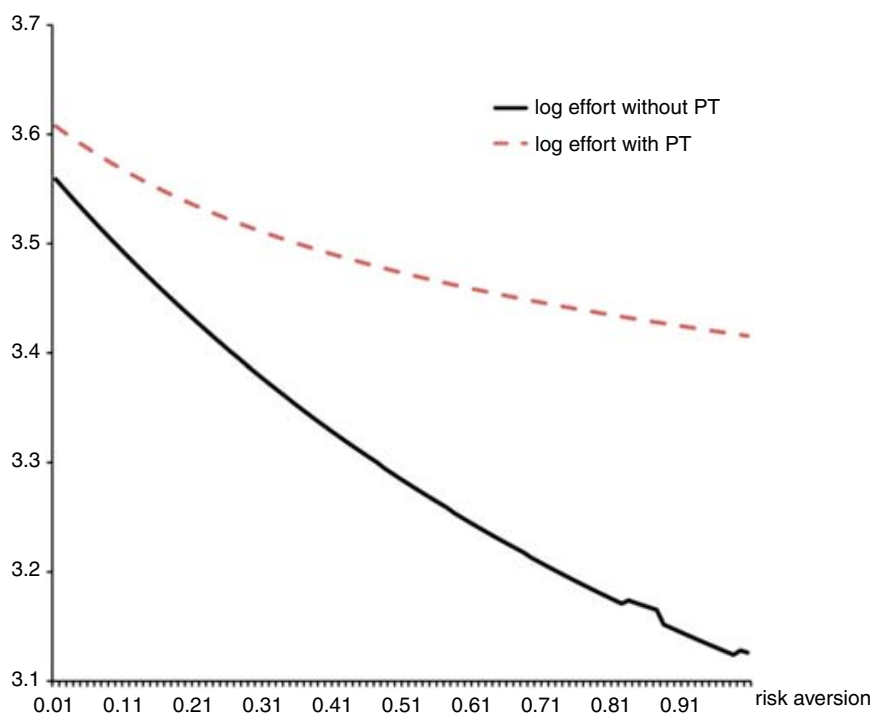

parameters in Table I. More risk-averse workers optimally put in lower effort under both regimes. However, with PT the effort is higher for all levels of risk aversion, and increasingly so for more risk-averse workers, resulting in a flattening of the risk aversion-effort link as compared to the case of no PT. Conveniently, the log effort-risk aversion link is linear to a good degree of approximation, permitting empirical estimation of this link with a simple linear regression.

\section{The experiment}

To test $H 1$ and 2 empirically, an experiment was conducted at a large Dutch university in the summer of 2011. The experiment consisted of the instructions session (five minutes), an unpaid training round (three minutes) and two paid-for rounds (five minutes each) with a five-minute break in between and afterwards, followed by a questionnaire on risk aversion and demographics, which took a few minutes to fill in. Altogether, the experiment lasted about 45 minutes, including the time it took to allocate the participants to their seats in the lab and to disburse the earnings. In total, 85 participants (54 women and 31 men, average age 23 years), all humanities and social science students, and never participated in experiments before, were recruited locally through advertisements put up in public areas. The participants were randomly assigned to the control group, receiving a fixed pay for participation in each round of the experiment, and the treatment group, receiving payment depending on their performance at the experimental task. The full details of the experiment, including the instructions to the participants and the forms, are available in the working paper version of this study (Zubanov, 2012).

The experimental task was to find the word on a specified page, line and position in a piece of printed text, and to enter it into the electronic form created for each round. The number of such entries per session is my measure of individual effort. Once the word was entered, a specially written computer program would determine whether the entry was correct and assign a payment to each participant in each round. Participants in the control group received a fixed pay of $€ 1.50$ per round, irrespective of the number 
of words they entered. Those in the treatment group were paid per word, earning $€ 0.20$ with probability 0.25 , and zero with probability 0.75 . This method of payment, implemented using a built-in random numbers generator, mimics the incentive pay scheme with multiplicative noise in which pay uncertainty increases with effort. To avoid satisficing, the participants were not informed how many words they entered correctly or, for the treatment group, how many of them were "winning" (i.e. carrying the $€ 0.20$ reward) until the very end of the experiment.

The payment rules for the treatment group participants differed by round. During the first paid-for round (round 1), they were paid for every winning word, as workers would be paid in the model without PT. During round 2, they got paid only if the number of winning words was equal or exceeded eight, as in the PT case studied in Section 2.1

Two differences between the experimental designs in my study and Cadsby et al.'s (2007, 2009) are worth mentioning. The first difference is in the experimental task. Word inputting, to my knowledge, has not been used in experimental work before. Yet, it suits the purpose of the experiment well, since performance on this task, while requiring effort, depends on ability less than anagram solving does, allowing more easily to abstract from the potentially confounding ability factor. The second difference lies in the payment rule, which was implemented in Cadsby et al. (2009) as a payment per anagram solved. Although the uncertainty in the time it takes to solve an anagram does result in the earnings uncertainty increasing with effort (see the concluding Section 7 for an example), paying per unit of task with a given probability, as is done in my experiment, is a more "clean" way of modeling the risk aversion-effort link, since it guarantees that earnings uncertainty will depend on effort, not ability.

Risk aversion, the key explanatory variable in this study, was measured using the menu of lottery choices developed in Holt and Laury (2002) at the end of the experiment. Participants were asked to choose between a safe (low variance) and a risky (high variance) options in ten lottery decisions in which the difference in expected values of the risky and the safe options increased progressively. The expected values of lottery payments were of a similar magnitude to the earnings in the experimental rounds. I use the number of chosen safe options as the measure of the degree of risk aversion in the statistical analysis to follow[4]. To incentivize participants to give honest answers, one of the ten lottery decisions was picked at random and the option chosen by a participant in that decision was played to him or her. Together with the winnings from this lottery and a show-up fee of $€ 1.50$, the average earnings in the control and treatment groups were $€ 6.3$ and $€ 7$, respectively.

\section{Preliminary results}

Table II reports summary statistics for the main variables of the experiment by group. The participants, 64 percent of whom were women and most in their mid 20's, exhibit an average attitude to risk close to neutrality. The average number of safe choices, about four, is somewhat lower than a mildly risk-averse 5.17 reported by Holt and Laury's (2002) experiment participants when confronted with lottery payments of a comparable monetary value[5]. There is considerable variation in the reported number of safe choices spanning from zero to seven in the control group, and from zero to nine in the treatment group.

The measure of effort I use is the total number of words entered (there were negligibly few incorrect entries). It averages at about 35 words for both groups in round 1 and then increases a little, to 36 in the control group and to 37 in the treatment group. 


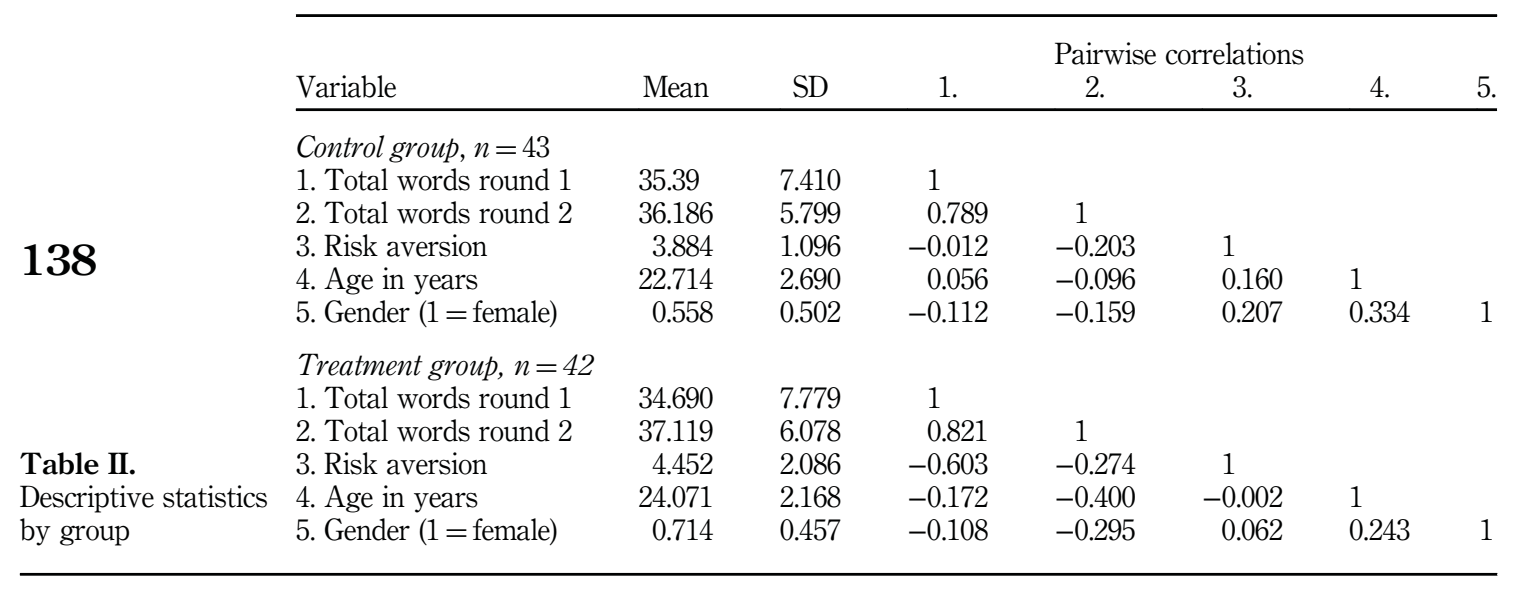

It shows correlations with risk aversion mostly consistent with my theory: negative $(-0.6)$ in the treatment group in the no-PT round 1, negative but weaker in the same group in the PT round $2(-0.27)$, and insignificant in the control group in round 1. A small but hard to ignore negative correlation between effort and risk aversion in the control group in round $2(-0.2)$ suggests that more risk averse people tend to get tired more quickly. The relationship between risk aversion and fatigue is outside my model but it may affect the estimation results as I explain below.

The similarity between average effort levels across groups and treatments is surprising, since it is only the treatment group whose performance was incentivized. The nature of the experimental task, which might have been regarded by some participants as mildly entertaining, may be one explanation; however, other factors closer to the interest of this study are likely to be at work as well. Thus, even though the allocation of participants into groups was random, the treatment group happens to be, on average, more risk-averse than control: 4.45 vs 3.88 , though this difference is statistically insignificant. There is also a large negative correlation between risk aversion and effort in the treatment group, consistent with the model. Further analysis will show that these two factors together have lowered the treatment group's average effort, bringing it close to that of the un-incentivized control group.

The improvement in the treatment group's average effort in round 2 relative to round 1, though consistent with the theory, is modest. While there were no cases of a steep decline in effort that would suggest a refusal to participate, fatigue might have negatively affected effort in round 2 . If fatigue is unrelated to risk aversion, its average effect will be part of the constant term without causing a bias to the regression estimates. However, looking at the correlations between risk aversion and effort in the control group, it seems that more risk-averse participants are more prone to fatigue. If this is the case, there will be a downward bias in the estimated effect of risk aversion on effort in round 2 , making $H 2$ harder, not easier, to verify.

\section{Econometric estimation issues}

In this section, I attempt to organize the experimental data with an empirical model capable of testing my hypotheses statistically. In principle, one could treat the firstorder conditions for the optimal effort levels with and without PT, $x_{m}^{*}$ and $x^{*}$, as 
implicit functions of risk aversion, and estimate their parameters from the data using nonlinear least squares. However, making statistical inferences from the results of this procedure will be difficult, since there is no structurally comparable utility maximization model for the control group as it receives no piece-rate incentives. A simpler and more direct way of testing the predictions of my model is to estimate a linear regression of log effort on risk aversion, allowing the regression parameters to vary between the treatment and control groups. The numerical solutions presented in Section 2.2 show that log-linear is indeed a good approximation for the effort-risk aversion link implied by the theory.

The regression equations I estimate are:

$$
\begin{aligned}
\ln \left(\text { total } \text { words }_{i}^{\text {round } 1}\right)= & \beta_{0}^{\text {round1 }}+\beta_{1}^{\text {round } 1} \times \text { treatment }_{i}+\beta_{2}^{\text {round } 1} \\
& \times \text { risk } \text { aversion }_{i}+\beta_{3}^{\text {round } 1} \times \text { treatment }_{i} \\
& \times \text { risk } \text { aversion }_{i}+\text { controls }_{i}+u_{i}^{\text {round } 1}, \\
\ln \left(\text { total } \text { words }_{i}^{\text {round2 }}\right)= & \beta_{0}^{\text {round } 2}+\beta_{1}^{\text {round } 2} \times \text { treatment }_{i}+\beta_{2}^{\text {round2 }} \\
& \times \text { risk } \text { aversion }_{i}+\beta_{3}^{\text {round } 2} \times \text { treatment }_{i} \\
& \times \text { risk } \text { aversion }_{i}+\text { controls }_{i}+u_{i}^{\text {round } 2},
\end{aligned}
$$

where $u_{i}^{\text {round1 }}$ and $u_{i}^{\text {round2 }}$ are the error terms. Coefficient $\beta_{3}^{\text {round1 }}$ is the text statistic for $H 1$; it measures the difference between the effect of risk aversion on effort in the treatment group, where it is supposed to matter, and the control group, where such link is not expected. Though not part of my theory, a correlation between risk aversion and effort may exist in the control group as well, for instance, due to cognitive ability affecting both effort and risk aversion (Dohmen et al., 2010). Coefficients $\beta_{2}^{\text {round1 }}, \beta_{2}^{\text {round2 }}$ measure this correlation in the respective rounds. Lastly, the expression $\beta_{1}^{\text {round1 }}+\beta_{3}^{\text {round1 }} \times$ risk aversion $i$ and its corresponding expression in round 2 measure the incentive effect as a function of risk aversion.

The test statistic for H2 (PT weakens the negative relationship between risk aversion and effort) is $\beta_{3}^{\text {round2 }}-\beta_{3}^{\text {round1 }}$, which is the difference between the effects of risk aversion on effort in round 2 (PT) and round 1 (no PT) estimated from Equations (9) and (8), respectively. Note that, given the adopted, linear, regression specification, $\beta_{3}^{\text {round2 }}-\beta_{3}^{\text {round1 }}$ also measures the effect of risk aversion on the effort increase caused by PT[6]. To calculate the standard error of this statistic, the variance-covariance matrix of coefficients $\beta_{3}^{\text {round2 }}$ and $\beta_{3}^{\text {round1 }}$ is needed. I obtain this matrix by estimating Equations (8) and (9) simultaneously, using Zellner's (1962) method of seemingly unrelated regressions. Since the regressors in both equations are identical, this method produces exactly the same results as ordinary least squares applied to each equation separately (Greene, 2003, pp. 343-344).

\section{Regression results}

Table III reports results for Equations (8) and (9) estimated jointly in specifications with and without controls, for all individuals together as well as for men and women separately. All controls are individually insignificant and their inclusion makes little difference to the estimates of main interest. Starting with the results on the entire 


\begin{tabular}{|c|c|c|c|c|}
\hline & \multicolumn{2}{|c|}{ All } & Men & Women \\
\hline $\begin{array}{l}\text { Dependent variable: } \log \text { total words round } 1 \\
\text { Treatment }\left(\beta_{1}^{\text {round1 }}\right)\end{array}$ & $\begin{array}{l}0.326^{* *} \\
(0.135)\end{array}$ & $\begin{array}{l}0.350^{* *} \\
(0.138)\end{array}$ & $\begin{array}{l}1.087^{* * * *} \\
(0.321)\end{array}$ & $\begin{array}{c}0.245 \\
(0.222)\end{array}$ \\
\hline Risk aversion $\left(\beta_{2}^{\text {round1 }}\right)$ & $\begin{array}{c}0.002 \\
(0.028)\end{array}$ & $\begin{array}{c}0.006 \\
(0.028)\end{array}$ & $\begin{array}{c}0.011 \\
(0.033)\end{array}$ & $\begin{array}{r}-0.009 \\
(0.050)\end{array}$ \\
\hline Treatment $\times$ Risk aversion $\left(\beta_{3}^{\text {round1 }}\right)$ & $\begin{array}{c}-0.079 * * \\
(0.032)\end{array}$ & $\begin{array}{c}-0.084^{* * * *} \\
(0.032)\end{array}$ & $\begin{array}{c}-0.258^{* * * *} \\
(0.076)\end{array}$ & $\begin{array}{r}-0.060 \\
(0.053)\end{array}$ \\
\hline Controls & no & yes & no & no \\
\hline $\begin{array}{l}\text { Dependent variable: log total words round } 2 \\
\text { Treatment }\left(\beta_{1}^{\text {round2 }}\right)\end{array}$ & $\begin{array}{c}0.017 \\
(0.108)\end{array}$ & $\begin{array}{c}0.072 \\
(0.106)\end{array}$ & $\begin{array}{l}0.636^{* * *} \\
(0.195)\end{array}$ & $\begin{array}{c}0.058 \\
(0.195)\end{array}$ \\
\hline Risk aversion $\left(\beta_{2}^{\text {round2 }}\right)$ & $\begin{array}{c}(0.100) \\
-0.033 \\
(0.022)\end{array}$ & $\begin{array}{c}(0.100) \\
-0.024 \\
(0.022)\end{array}$ & $\begin{array}{c}-0.036^{*} \\
(0.020)\end{array}$ & $\begin{array}{c}(0.15) \\
-0.011 \\
(0.044)\end{array}$ \\
\hline Treatment $\times$ Risk aversion $\left(\beta_{3}^{\text {round2 }}\right)$ & $\begin{array}{c}0.006 \\
(0.025)\end{array}$ & $\begin{array}{c}-0.003 \\
(0.025)\end{array}$ & $\begin{array}{c}-0.128^{* * *} \\
(0.046)\end{array}$ & $\begin{array}{r}-0.008 \\
(0.046)\end{array}$ \\
\hline Controls & no & yes & no & no \\
\hline $\begin{array}{l}\text { Incentive effect for the average individual, ro } \\
\beta_{1}^{\text {round1 }}+\beta_{3}^{\text {round1 }} \times \text { average risk aversion }\end{array}$ & $\begin{array}{l}\text { und } 1 \\
-0.005 \\
(0.044)\end{array}$ & $\begin{array}{c}0.001 \\
(0.046)\end{array}$ & $\begin{array}{c}0.087 \\
(0.077)\end{array}$ & $\begin{array}{r}-0.013 \\
(0.053)\end{array}$ \\
\hline $\begin{array}{l}\text { Incentive effect for the average individual, ro } \\
\beta_{1}^{\text {round2 }}+\beta_{3}^{\text {round2 }} \times \text { average risk aversion }\end{array}$ & $\begin{array}{l}\text { und } 2 \\
0.042 \\
(0.035)\end{array}$ & $\begin{array}{c}0.059^{*} \\
(0.035)\end{array}$ & $\begin{array}{l}0.141^{* * * *} \\
(0.047)\end{array}$ & $\begin{array}{c}0.025 \\
(0.046)\end{array}$ \\
\hline $\begin{array}{l}\text { Difference in the risk aversion's effect on eff } \\
\beta_{3}^{\text {round } 2}-\beta_{3}^{\text {round1 }}\end{array}$ & $\begin{array}{c}\text { rt with and } \\
0.085^{* * *} \\
(0.018)\end{array}$ & $\begin{array}{l}\text { out } P T \\
0.081^{* * * *} \\
(0.018)\end{array}$ & $\begin{array}{l}0.131^{* * * *} \\
(0.048)\end{array}$ & $\begin{array}{c}0.052^{*} \\
(0.029)\end{array}$ \\
\hline
\end{tabular}

Table III.

Regression results for $H 1$ and $H 2$
Notes: Standard errors for linear combinations of regression coefficients; are calculated using the coefficient variance-covariance matrices; estimated with seemingly unrelated regressions. *******:Significant at 10,5 and 1 percent levels, respectively

sample, there is no significant link between risk aversion and effort in the control group (coefficients $\beta_{2}^{\text {round1 }}$ and $\beta_{2}^{\text {round2 }}$ ), which was expected. In the treatment group, however, the link is negative. The test statistic for $H 1, \beta_{3}^{\text {round1 }}$, is estimated at about -0.08 on the entire sample, and is significant, supporting the prediction that effort under a linear incentive pay scheme with multiplicative noise decreases with risk aversion. The test statistics for $H 2, \beta_{3}^{\text {round2 }}-\beta_{3}^{\text {round1 }}$, is also about 0.08 in magnitude and is strongly significant. Hence, the existence of the PT weakens the negative risk aversion-effort link observed without it. This link weakens because more risk-averse participants increase their effort by a greater margin (8 percent with a unit increase in risk aversion, or about 0.6 of its standard deviation) than do less risk-averse ones. Recalling that more risk-averse participants may have reduced their effort because of experiencing greater fatigue, this estimate is likely to be a conservative one.

Having confirmed both hypotheses, let us examine the implications of the negative risk aversion-effort link on the effect of incentive pay on effort. Although the treatment dummy itself has a large positive and significant coefficient in round $1\left(\beta_{1}^{\text {round1 }}\right)$, the treatment effect is greatly reduced by the negative risk aversion-effort link specific to the treatment group. In fact, the treatment effect for the average participant in round 1 
$\left(\beta_{1}^{\text {round } 1}+\beta_{3}^{\text {round } 1} \times\right.$ average risk aversion in Table III) is almost zero, suggesting that the negative risk aversion-effort link is powerful enough to bring performance gains from incentive pay to naught. The situation improves, albeit slightly, in round 2, where PT is introduced. In round 2, the treatment effect for the average participant is 0.042-0.059 and is on the brink of statistical significance. It is, again, a conservative estimate, since the treatment group, more risk-averse on average, may have experienced greater fatigue than the control group. Thus, there are signs that PT could restore the efficiency of incentive pay schemes.

Focussing on the results for men and women separately, there is a large negative risk aversion - effort link for men under multiplicative noise in the absence of PT $(-0.258$, round 1), which greatly weakens the incentive effect for the average individual, putting it at an insignificant 0.087 . The introduction of PT in round 2 restores the strength of monetary incentives for men to a significant 0.141 by cancelling the effect of risk aversion on effort by about half. The results for women, though qualitatively similar to men's, are much weaker in magnitude.

\section{Discussion, preliminary conclusions and further research}

Let us take stock of the findings so far, relate them to the wider literature and the practice of personnel management, and outline possible future research directions. The model presented in my study predicts that risk aversion will affect individual choice of effort in the multiplicative noise case, when the noise to output and effort are not independent (H1). Only limited scholarship exists on this case. Yet, it is important practically, applying whenever each individual instance of effort succeeds with a certain probability, such as in the sales agent example in the introduction.

The data from the real-effort laboratory experiment featured in this study support $H 1$, especially so for male participants, although further experiments, carried out on a larger scale, are necessary to strengthen its empirical foundations. The finding that effort tends to decrease with risk aversion will inform personnel managers that efficiency gains from incentive pay schemes in their firms may be limited by workers' dislike of risk. In fact, these gains are very low for the average participant in our experiment. This finding speaks to the literature on the determinants of incentive pay's adoption by firms, especially with respect to non-managerial employees (Parent, 2002; Barth et al., 2008; Bryson et al., 2012), suggesting that workers' risk aversion could be one of such determinants, at least in the short run when sorting of people of different risk preferences into jobs with different exposure to risk cannot be completed.

The second hypothesis featured in this study was that PT should weaken the link between risk aversion and effort, at least for the workers who will still participate, because PT will cause more risk-averse workers to put in more additional effort. This hypothesis has been supported by the experimental data as well. By implication, the introduction of PT may restore part of the incentive pay effect on performance, which has been seen in the data, too. These findings speak to the literature on the use of PTs in incentive pay schemes, providing an additional explanation to the existence of this popular practice. The lesson for management is that PTs can be an effective tool for motivating workers, especially those economizing on effort because of being risk-averse.

Much research is still needed on the risk aversion - effort link in situations, summarized by the term "multiplicative noise," when the noise to output is not independent of effort. Such situations abound in practice and may even include cases 
when reward is certain and given per each attempt, as in Cadsby et al's $(2007,2009)$ experiments. Here I will outline two extensions to my model to motivate further research on the topic, both drawing on the original sales agent story. In the first extension, let the length of time per customer contact vary randomly following an exponential distribution. Then the number of customers that can be contacted in a working day follows a Poisson distribution, with its variance equal to the mean equal to the expected number of customer contacts per day. That is, even when paid per contact rather than per deal, agents who will make more contacts will face higher earnings uncertainty, which will give rise to a risk aversion - effort link not unlike the one studied in this paper.

In the second extension, let the probability of success in each contact increase with effort rather than stay constant. The agent will then have to decide on the amount of effort per contact as well as on the number of contacts to make. Intuition supported with some back-of-the-envelope calculus suggests that the more risk-averse will make fewer contacts but spend more effort per contact, whereas the more risk loving will do the opposite. While the link between risk aversion and total effort is now less clear, the influence risk preferences seem to make on the work styles and outcomes is worth studying further.

Finally, a larger question - are PTs economically efficient as well as effective? needs to be addressed. The utility loss caused by PT requires compensation to keep the workers employed at the firm, the more the higher their risk aversion. In my simple model, a risk-neutral firm could weaken the negative risk aversion-effort link more cheaply by replacing a random commission $\gamma$ with its expectation, $\bar{\gamma}$, so as to remove the multiplicative noise component from incentive pay. Doing so, however, may invite dysfunctional behavioral response in a more complicated setting, whereby workers would maximize throughput compromising on quality. A more systematic exploration of behavioral responses and efficiency implications of different incentive schemes with multiplicative noise awaits further research.

\section{Notes}

1. The experimental studies by Sloof and van Praag $(2008,2010)$ are close to mine in focus and method, but they are done with the noise additive to effort.

2. The theoretical results for the power utility function, another popular choice in the literature (e.g. Bellemare and Shearer, 2010), available from the author, are weaker but still hold for reasonable values of the decision parameters.

3. The Matlab code for the numerical solutions is available on request.

4. One could also recover intervals of the direct measure of risk aversion, $\delta$, consistent with the stated lottery preferences and use the midpoint in each interval as an alternative measure of risk aversion. The results with this measure (available on request) are qualitatively the same.

5. The average number of safe choices in the Holt and Laury experiment was calculated based on the results reported Table III in their 2002 article.

6. The equation linking effort increase to risk aversion is: $\ln \left(\frac{\text { total words round } 2}{\text { total words round } 1}\right)_{i}=\tilde{\beta}_{0}+\tilde{\beta}_{1} \times$ treatment $_{i}+\tilde{\beta}_{2} \times$ risk aversion ${ }_{i}+\tilde{\beta}_{3} \times$ treatment $_{i} \times$ risk aversion $_{i}+$ controls $_{i}+\tilde{u}_{i}$, where $\tilde{x}=x^{\text {round2 }}-x^{\text {round1 }}$, which is derived by subtracting Equations (8) from (9) part by part. The treatment effect is $\tilde{\beta}_{3}=\beta_{3}^{\text {round2 }}-\beta_{3}^{\text {round1 }}$. 


\section{References}

Baker, G. (1992), "Incentive contracts and performance measurement", Journal of Political Economy, Vol. 100 No. 3 , pp. 598-614.

Baker, G. and Jorgensen, B. (2003), "Volatility, noise, and incentives”, unpublished working paper.

Barth, E., Bratsberg, B. and Haegeland, T. (2008), "Who pays for performance?”, International Journal of Manpower, Vol. 29 No. 1, pp. 8-29.

Basu, A.K., Lal, R., Srinivasan, V. and Staelin, R. (1985), "Salesforce compensation plans: an agency theoretic perspective", Marketing Science, Vol. 4 No. 4, pp. 267-291.

Bellemare, C. and Shearer, B. (2010), "Sorting, incentives and risk preferences: evidence from a field experiment”, Economics Letters, Vol. 108, pp. 345-348.

Bryson, A., Freeman, R., Lucifora, C., Pellizzari, M. and Perotin, V. (2012), "Paying for performance: incentive pay schemes and employees' financial participation”, Discussion Paper No. 1112, CEP, London.

Cadsby, B., Song, F. and Tapon, F. (2007), "Sorting and incentive effects of pay-for -performance: an experimental investigation”, Academy of Management Journal, Vol. 50 No. 2, pp. 387-405.

Cadsby, B., Song, F. and Tapon, F. (2009), "The impact of risk aversion and stress on the incentive effect of performance pay", unpublished working paper, Guelph, ON.

Chung, D.J., Steenburgh, T. and Sudhir, K. (2014), "Do bonuses enhance sales productivity? A dynamic structural analysis of bonus-based compensation plans", Management Science, Vol. 33 No. 2, pp. 165-187.

Coughlan, A.T. and Joseph, K. (2012), "Sales force compensation: research insights and research potential", in Lilien, G. and Grewal, R. (Eds), Handbook of Business-to-Business Marketing, Edward Elgar Publishing, Cheltenham, pp. 473-495.

Dohmen, T., Falk, A., Huffman, D. and Sunde, U. (2010), "Are risk aversion and impatience related to cognitive ability?”, American Economic Review, Vol. 100 No. 3, pp. 1238-1260.

Gaba, A. and Karla, A. (1999), "Risk behavior in response to quotas and contests", Marketing Science, Vol. 18 No. 3, pp. 417-434.

Greene, W.H. (2003), Econometric Analysis, 5th ed., Prentice Hall, Upper Saddle River, New Jersey, NJ.

Grund, C. and Sliwka, D. (2010), "Evidence of performance pay and risk aversion", Economics Letters, Vol. 102, pp. 8-11.

Holmstrom, B. and Milgrom, P. (1987), "Aggregation and linearity in the provision of intertemporal incentives", Econometrica, Vol. 55 No. 2, pp. 303-328.

Holt, C.A. and Laury, S.K. (2002), "Risk aversion and incentive effects", American Economic Review, Vol. 92 No. 5, pp. 1644-1655.

Lazear, E.P. (2000), "Performance pay and productivity", American Economic Review, Vol. 90 No. 5, pp. 1346-1361.

Lazear, E.P. and Gibbs, M. (2009), Personnel Economics in Practice, 2nd ed., John Willey \& Sons, New York, NY.

Lo, H.F., Ghosh, M. and Lafontaine, F. (2011), "The incentive and selection roles of sales force compensation contracts", Journal of Marketing Research, Vol. 48 No. 4, pp. 781-798.

Pappas, J.M. and Flaherty, K.E. (2006), "The moderating role of individual-difference variables in compensation research", Journal of Managerial Psychology, Vol. 21 No. 1, pp. 19-35.

Parent, D. (2002), "Performance pay in the United States: its determinants and effects", in Brown, M. and Heywood, J.S. (Eds), Paying for Performance: An International Comparison, M. E. Sharpe Inc, Armonk, NY, pp. 17-51. 
Prendergast, C. (2000), "What trade-off of risk and incentives", American Economic Review Papers and Proceedings, Vol. 90, pp. 421-425.

Raju, J.A. and Srinivasan, V. (1996), "Quota-based compensation plans for multiterritory heterogeneous salesforces”, Management Science, Vol. 42 No. 10, pp. 1454-1462.

Sloof, R. and van Praag, C.M. (2008), "Performance measurement, expectancy and agency theory: an experimental study", Journal of Economic Behavior and Organization, Vol. 67 Nos 3-4, pp. 794-809.

Sloof, R. and van Praag, C.M. (2010), "The effect of noise in a performance measure on work motivation: a real effort laboratory experiment”, Labour Economics, Vol. 17, pp. 751-765.

Vroom, V. (1964), Work and Motivation, Wiley, San Francisco.

Zellner, A. (1962), "An efficient method of estimating seemingly unrelated regression equations and tests for aggregation bias", Journal of the American Statistical Association, Vol. 57 No. 298, pp. 348-368.

Zubanov, N. (2012), "Risk aversion and effort in an incentive pay scheme with multiplicative noise: theory and experimental evidence", ERIM Report Series Research in Management, Rotterdam, available at: www.hdl.handle.net/1765/32031

\section{About the author}

Nikolay Zubanov is a Professor of Management Science and Strategy at the Goethe University Frankfurt. He obtained his $\mathrm{PhD}$ in 2007 from the University of Birmingham (UK) and held academic positions at the Tilburg University and Erasmus University Rotterdam in The Netherlands. Professor Nikolay Zubanov can be contacted at: zubanov@econ.uni-frankfurt.de 